\title{
Español como lengua extranjera en Brasil: cómo enseñar colocaciones con las TIC
}

Ivonne J. de Mogendorff ${ }^{\mathrm{i}}$

\section{RESUMEN}

Este trabajo se incluye en el ámbito de la Fraseología, y trata de la enseñanzaaprendizaje de las colocaciones (CORPAS PASTOR, 1996; KOIKE, 2000) formadas por verbo+sustantivo en las que una de sus partes difiere entre el español y el portugués, como "mirar televisión" y "assistir televisão". Estas combinaciones léxicas no se trabajan de forma clara y explícita y, por desconocimiento, los aprendices brasileños de español las transfieren de su lengua materna a esta lengua en la producción oral y escrita. Para guiar y promover en los aprendices, una práctica significativa en el uso de de las tecnologías de la información y la comunicación (TIC), elaboramos un material didáctico digital con colocaciones divergentes destinado a estudiantes brasileños de la Educación Básica, con un nivel de español cercano a A2.

Palabras clave: Lengua Española; Colocaciones; Verbo+sustantivo; EnseñanzaAprendizaje; TIC.

\section{RESUMO}

Este trabalho insere-se no âmbito da Fraseologia, e trata do ensino-aprendizagem das colocações (CORPAS PASTOR, 1996; KOIKE, 2000) formadas por verbo+substantivo quando uma de suas partes difere entre o espanhol e o português, como "mirar televisión" e "assistir televisão". Estas combinações léxicas não se trabalham de forma clara e explícita e, por desconhecimento, os aprendizes brasileiros de espanhol as transferem de sua língua materna para esta língua na produção oral e escrita. Para orientar e promover nos aprendizes, uma prática significativa no uso das tecnologias da informação e comunicação (TIC), elaboramos um material didático digital com colocações divergentes destinado a estudantes brasileiros da Educação Básica, com um nível de espanhol próximo de A2.

Palavras-chave: Língua espanhola; Colocações; Verbo+substantivo; Ensinoaprendizagem; TIC.

\footnotetext{
${ }^{\text {i }}$ Licenciatura em Letras Espanhol (UFRGS, 2005), Mestrado em Literaturas Estrangeiras Modernas: Literaturas de Língua Espanhola (UFRGS, 2009) e Doutorado em Lexicografia, Terminologia e Tradução: Relações Textuais (UFRGS, 2019). Atualmente é professora de Língua Espanhola do Colégio de Aplicação da Universidade Federal do Rio Grande do Sul. https://orcid.org/0000-0001-9005-1802 | ivonne.jordan@hotmail.com
} 


\section{INTRODUCCIÓN}

El presente trabajo se incluye en el ámbito de los Estudios del Léxico, más específicamente, en el área de la Fraseología y se refiere a la enseñanza y práctica digital de las colocaciones formadas por verbo+sustantivo divergentes entre el español y el portugués, a estudiantes brasileños de la Educación Básica con un nivel cercano al de un Usuario Básico A2, de acuerdo con los Niveles Comunes de Referencia del Marco Común Europeo de Referencia para las lenguas (CONSEJO DE EUROPA, 2002).

El uso de las tecnologías informáticas ha transformado el aula de lengua extranjera y, como consecuencia, se ha integrado en la labor docente. Actualmente, es improbable que el profesor imparta clase sin usar la red para recabar información, crear materiales motivadores o incluirla en las actividades didácticas diarias. Esta expansión fuera del espacio físico de la sala de aula no lleva siempre aparejado el uso de soportes tecnológicos apropiados y actualizados debido a que el profesor depende de las posibilidades que cada escuela ofrece en términos de pantallas, ordenadores, cañones de proyección y por cierto, redes wifi y conexiones eléctricas. En nuestro caso, enseñar español a alumnos adolescentes brasileños de la enseñanza regular implica practicar lenguas por medio de actividades diversificadas que incluyan el uso de las TIC para una práctica real, valiéndonos de los diversos recursos que la red ofrece de acceso a información y a interacción (MOREIRA KENSKI, 2008).

Las TIC poseen un gran potencial para desarrollar la competencia comunicativa digital de los estudiantes de español como lengua extranjera (ELE) que abarca desde aprender los sistemas lingüísticos básicos, practicar las habilidades comunicativas o llevar a cabo los procedimientos para realizar una tarea final integrando los conocimientos aprendidos (PUJOLÁ, 2016).

Cassany (2012) aclara que los jóvenes actualmente se muestran muy activos digitalmente en los espacios informales, pero que esto no significa que al navegar por internet sepan buscar materiales o jerarquizar la calidad de los mismos. Es necesario que los alumnos realicen una práctica significativa para que realmente puedan utilizar esta tecnología. Para Pujolà (2016), los jóvenes utilizan las TIC de forma superficial, dentro del aprendizaje formal, por falta de conocimientos digitales. Como docentes de ELE precisamos guiar y estimular la lectura y escritura digital del aprendiente propiciando 
prácticas que favorezcan el aprendizaje de la lengua española, al mismo tiempo que los alumnos se capacitan frente a las múltiples posibilidades que la red ofrece.

Por ello, Cassany explica que

\begin{abstract}
el ordenador sólo ofrece las potencialidades (...) para acceder a más datos y comunicaciones más sofisticadas; lo que genera aprendizaje e inteligencia es la práctica cognitiva y social de ejecutar estas potencialidades en contextos reales y significativos hasta el punto de poder apropiarnos de esas herramientas y de convertirlas en instrumentos básicos para nuestra vida. (CASSANY, 2012, p. 40-41)
\end{abstract}

El alumno no se vuelve tecnológicamente competente sólo por hacer uso del ordenador, se hace necesario aprender a usar sus recursos de forma positiva.

Moreira Kenski (2008) aclara que los adelantos tecnológicos reorientan a una lectura de otro tipo de textos o imágenes. El texto electrónico presenta recursos que el texto en papel no tiene. Se pueden establecer variadas relaciones, cruzar informaciones y compararlas en muy poco tiempo. Las tecnologías permiten el vínculo entre la escuela y el mundo fuera de la escuela acercando estas vivencias a los alumnos.

Estamos preparando el alumnado para un futuro que exige, por cierto, el dominio de la literacidad digital entendida como un conjunto de habilidades sociales e individuales, imprescindibles para circular por el mundo digital (DUDENEY; HOCKLY; PEGRUM, 2016). Se debe capacitar a los alumnos a usar eficazmente esta tecnología que implica desde encontrar recursos a través de múltiples plataformas hasta transmitir ideas e informaciones.

Dudeney, Hockly y Pegrum (2016) concluyen que enseñar lenguas únicamente a través de la literacidad impresa es engañar a nuestros alumnos en su presente y en sus necesidades futuras. Para estos autores existe la creencia de que los jóvenes son tecnológicamente competentes, pero esta percepción se enfrenta a una serie de factores como posición socioeconómica, nivel de educación y lengua, entre otros y conlleva el uso adecuado de la tecnología para entretenimiento y prácticas sociales, sin embargo, para objetivos educacionales que presuponen una comprensión crítica, estos jóvenes dependen de la orientación de los profesores.

Como profesores de ELE consideramos que las TIC se alinean con nuestra labor docente y con el papel del alumno como responsable por su propio aprendizaje. En base a todo esto hemos elaboramos una propuesta didáctica valiéndonos del uso de las TIC para la enseñanza y práctica de las colocaciones de verbo+sustantivo divergentes entre 
el español y el portugués. Se trata de una novela digital en lengua española, con temas afines a los intereses del público meta, acompañada de ejercicios contextualizados. Este material, al mismo tiempo que promueve el aprendizaje de las colocaciones, favorece el uso del aula como espacio de aprendizaje digital "que requiere el acceso a la red para buscar, usar y elaborar información pertinente" (CASSANY 2012, p. 255). De esta forma, se espera promover de forma conjunta la competencia digital y el desarrollo de la competencia colocacional de los aprendientes.

\section{¿POR QUÉ ENSENAR COLOCACIONES DE VERBO+ SUSTANTIVO?}

En relación a la enseñanza de ELE, hemos observado una preferencia generalizada en priorizar la enseñanza-aprendizaje de las estructuras gramaticales frente al aprendizaje del léxico. Sin embargo, no alcanza con aprender la gramática y listas de palabras sin ningún uso funcional para comunicarse en la nueva lengua; hay que dedicar más tiempo a enseñar el léxico de forma contextual visto su relevancia para el desarrollo de la competencia comunicativa. En 1972, Hymes (citado en RICHARDS y RODGERS, 1998) formuló esta teoría que incluye los conocimientos lingüísticos, y también, las habilidades para emplear la lengua apropiadamente según el contexto. Por tanto, desde esta perspectiva, el proceso de adquisición del léxico implica ejercitar, de forma completa, todas las habilidades que constituyen la competencia comunicativa y posibilitar que el aprendiente tenga la oportunidad de una práctica consciente y activa.

Cuando el hablante nativo se expresa utiliza de forma intuitiva, desde palabras simples a combinaciones de vocablos con un variable grado de estabilidad. Este último grupo, objeto de estudio de la Fraseología está compuesto por unidades fraseológicas (UFs). Corpas Pastor las define como:

unidades léxicas formadas por más de dos palabras gráficas en su límite inferior, cuyo límite superior se sitúa en el nivel de la oración compuesta. Dichas unidades se caracterizan por su alta frecuencia de uso, y de coaparición de sus elementos integrantes; por su institucionalización, entendida en términos de fijación y especialización semántica; por su idiomaticidad y variación potenciales; así como por el grado en el cual se dan todos estos aspectos en los distintos tipos. (CORPAS PASTOR, 1996, p. 20) 
Corpas Pastor (1996) presenta una clasificación de las UFs de la lengua española a partir de cómo aparece el enunciado en el discurso. Esta autora (1996, p. 51) entiende el concepto de enunciado como "una unidad de comunicación mínima que corresponde generalmente a una oración simple o compuesta, pero también puede estar compuesta de un sintagma o una palabra". El enunciado puede ser incompleto o completo y conforme su fijación en la norma, en el sistema o en el habla, de acuerdo con la tricotomía de Coseriu (1980) la autora clasifica las UFs en tres esferas.

La esfera I comprende las COLOCACIONES, enunciados incompletos fijos en la norma, sintagmas que han desarrollado algún grado de fijación debido a su uso como “año fiscal” y “celebrar una reunión”. La esfera II reúne enunciados incompletos fijos en el sistema que generalmente funcionan como elementos oracionales con una unidad de significado, son las locuciones. Y por último, la esfera III está formada por enunciados completos con fijación y abarca actos del habla que pertenecen al acervo social-cultural de la comunidad hablante como paremias, citaciones, refranes y fórmulas rutinarias.

Conforme la clasificación de Corpas Pastor (1996, p. 51) las colocaciones son UFs pertenecientes a la esfera I.

La primera esfera de nuestra clasificación comprende colocaciones, es decir,
unidades fraseológicas que, desde el punto de vista del sistema de la lengua,
son sintagmas completamente libres, generados a partir de reglas, pero que, al
mismo tiempo, presentan cierto grado de restricción combinatoria
determinada por el uso (cierta fijación interna) [...] Presentan fijación externa
analítica, al ser empleadas como combinaciones de determinadas unidades
léxicas para el análisis ya establecido del mundo, frente a otras unidades
teóricamente posibles en la misma medida. (CORPAS PASTOR, 1996, p. 53)

Las colocaciones se diferencian de las combinaciones libres de palabras porque tienen una cierta fijación interna, y de las locuciones porque son combinaciones con fijación en la norma y no en el sistema (COSERIU, 1980). Por ello, al utilizar frecuentemente ciertas estructuras, una comunidad de hablantes termina por fijar determinadas combinaciones y las consagra como las más usuales revelando, de esta manera, la importancia del aprendizaje de los fraseologismos en el desarrollo de la competencia comunicativa.

Las colocaciones son idiosincráticas y, por tanto, cada comunidad elige, establece y fija por el uso sus propias combinaciones, lo que representa una dificultad para el aprendiente. Como son fácilmente comprensibles, porque son total $\mathrm{o}$ 
parcialmente transparentes, los alumnos las comprenden perfectamente, pero en la producción, si el alumno desconoce las UFs utilizadas en español, hace la transferencia de su lengua materna, en este caso el portugués de Brasil, a la lengua española y esto representa un obstáculo en el aprendizaje. En portugués decimos falar a verdade y si el aprendiente no sabe cuál es la combinación correcta en español, influenciado por su lengua materna, optará por usar hablar la verdad, siendo que la colocación en español es "decir la verdad".

Podemos dividir las colocaciones en dos categorías: las colocaciones gramaticales como "acordarse de", "caer en", que son de naturaleza sintáctica, compuestas de una construcción de verbo con complemento preposicional de régimen y las colocaciones léxicas. Nuestra propuesta didáctica se abocará sobre las colocaciones léxicas (KOIKE, 2000) que se dividen en colocaciones simples, formadas por dos unidades léxicas simples y colocaciones complejas, formadas por una sola unidad léxica y una UF como "levantar castillos en el aire". Visto que las colocaciones simples son las más utilizadas en los niveles iniciales decidimos dedicarnos a su práctica.

Las colocaciones léxicas simples, conforme la taxonomía de Koike (2000), presentan los siguientes aspectos formales y semánticos: coocurrencia frecuente de dos lexemas, restricciones combinatorias impuestas por el uso tradicional, composicionalidad formal que admite cierta flexibilidad combinatoria, vínculo propio entre los dos lexemas, relación típica entre sus componentes y precisión semántica de la combinación.

Írsula (1994) explica que las colocaciones pueden estar formadas por las siguientes combinaciones: verbo+sustantivo, adjetivo+sustantivo, verbo+adverbio y adverbio+adjetivo. De acuerdo con este autor (ídem, p. 281), "las colocaciones sustantivo-verbales representan el grupo más numeroso y que mayor influencia tiene en la comunicación lingüística" y, por ello, este material se elaboró con base en esta categoría.

A partir de estas consideraciones, el objetivo de este trabajo es presentar una propuesta didáctica mediante el uso de las TIC, para la enseñanza-aprendizaje de las colocaciones simples de verbo+sustantivo. Esta propuesta es un recurso que se les ofrece a los profesores de ELE de Brasil para trabajar las colocaciones de manera eficaz y entretenida estimulando y guiando a los aprendices adolescentes de ELE en el uso de 
recursos de la red para aprender la lengua española porque, como señala Pujolà (2016, p. 119), "es impensable en la actualidad planificar cualquier proceso didáctico en el aprendizaje de lenguas sin tener en cuenta de manera central o tangencial el uso de las TIC" como herramienta en el proceso de enseñanza de idiomas.

\section{METODOLOGÍA PARA LA SELECCIÓN DE LAS COLOCACIONES DE VERBO+SUSTANTIVO}

El corpus de colocaciones de verbo+sustantivo en lengua española fue escogido a partir de producciones orales y escritas de estudiantes de los años finales de la Educación Básica, y extraídas de textos periodísticos de El País (Uruguay) y La Nación (Argentina). En las colocaciones se prefirió elegir la variedad diatópica del español de la región del Río de la Plata (Uruguay y Argentina) ya que nuestra práctica docente se realiza en una escuela de Rio Grande do Sul, estado vecino de estos países.

En cuanto a la correlación entre el nivel de los alumnos y las colocaciones seleccionadas, percibimos que algunos estudios priorizan los criterios de frecuencia en vista de que el vocabulario frecuente representa un porcentaje considerable del vocabulario utilizado en los textos. Por otro lado, García Salido (2017) puntualiza que los estudios no han corroborado que un sistema de nivelación basado en la frecuencia "se corresponda con el grado de dificultad que presentan las colocaciones a los aprendices" (GARCÍA SALIDO, 2017, p. 46). Por ese motivo entendemos que influyen otros factores y, por tratarse de alumnos escolares, consideramos que, en ocasiones, una colocación no tan frecuente puede resultar productiva para el aprendiz.

En esta primera etapa encontramos dificultades para inferir si algunas de las combinaciones se ajustaban de forma rigurosa a los criterios teóricos sobre las colocaciones. Por ese motivo, nos valimos igualmente de las consideraciones de Sánchez Rufat (2010) y de la categorización de colocaciones prototípicas y no prototípicas de Higueras García (2006).

A partir de ello y basándonos en el marco teórico expuesto anteriormente, usamos los siguientes criterios para seleccionar las colocaciones:

a) Estar formada por una base y un colocativo de verbo+sustantivo o sustantivo+verbo pudiendo incluir una palabra gramatical (preposición); 
b) La base o el colocativo de la colocación en lengua española (o ambos) debe ser divergente de una o ambas partes de la colocación en portugués de Brasil. Por ejemplo: ver una película/assistir a um filme, escuchar una canción/escutar uma música, prender la televisión /ligar a televisão.

A continuación, para comprobar si se trataba realmente de una colocación, analizamos las combinaciones seleccionadas por medio de dos enfoques: semántico y estadístico.

Para el enfoque semántico nos apoyamos en el diccionario monolingüe combinatorio Redes (BOSQUE, 2010) que describe las restricciones léxicas que hay entre predicados y argumentos verificando la existencia de relaciones de tipicidad entre los elementos de la colocación.

Posteriormente, examinamos estas combinaciones mediante el enfoque estadístico. El mismo se realizó por medio de la aplicación de consulta en línea del CORPES XXI (RAE) que presenta los datos estadísticos de las coapariciones de una palabra con un término delimitado para, así, comprobar su institucionalización.

Se aplicaron los siguientes requisitos sobre el conjunto de colocaciones seleccionadas:

a) Figurar en el Diccionario Redes o, de no estar incluida en dicha obra, justificar su presencia;

b) Tener una frecuencia mínima de $10 \%$ en las coapariciones del CORPES XXI o, en caso contrario, justificar su presencia.

Finalmente seleccionamos 50 colocaciones basándonos en diversos factores como frecuencia, utilidad, disponibilidad y nuestra experiencia didáctica como profesores de ELE con el objetivo de obtener un grupo de colocaciones divergentes rentable comunicativamente.

Presentamos una muestra de algunas de las colocaciones seleccionadas: apagar el celular/desligar o celular, arrancar el coche/ligar o carro, arreglar el cuarto/arrumar o quarto, bajar del ómnibus/ descer do ônibus, contestar una pregunta/responder uma pregunta, dar una mirada/dar uma olhada, darse una ducha/tomar banho, doblar la 
esquina/ virar a esquina, dormir la siesta/fazer a sesta, tirar uma soneca, enamorarse de un/a chic@/apaixonar-se por um/a garot@, esperar el turno lesperara suavez.

\section{UNA PROPUESTA DIDÁCTICA PARA ENSEÑAR COLOCACIONES CON LAS TICS}

La red posibilita trabajar contenidos lingüísticos de forma diversificada, y el profesor precisa estimular la atención del aprendiente, por medio de tareas de su interés, como forma de que el aprendiz se involucre activamente. También es preciso guiar al alumno para que se convierta en responsable de su propio aprendizaje. Cassany (2012, p. 30) puntualiza que "muchos maestros comentan que hoy los chicos quizá tengan acceso a más información que antes, pero tienen más dificultad para construir conocimiento con ella".

Para una práctica efectiva es importante reflexionar que la motivación del alumno estimula su interacción con los materiales con los que trabaja y que al mismo tiempo, es preciso promover la autonomía del aprendiz con vistas a su aprendizaje actual y futuro. Por ese motivo, cuando se elabora la actividad es fundamental que sea interesante y llame la atención del público meta fomentando la metacognición sobre el aprendizaje.

Fernández López (2010) explica que las tareas en el aprendizaje de las lenguas son:

\footnotetext{
Actividades concretas, reales o verosímiles, acciones de la vida cotidiana no sólo académicas-, de interés para los alumnos, que se realizan en clase, o desde la clase, y que como ejes de la unidad didáctica, dinamizan los procesos de comunicación, y de aprendizaje y llevan a aprender todo lo necesario para poder realizarlas en la nueva lengua. (FERNÁNDEZ LÓPEZ, 2010, p. 7)
}

Por tanto, el objetivo de las clases de lengua extranjera es desarrollar la competencia comunicativa lingüística de los alumnos por medio de tareas significativas. "Los adolescentes, como grupo de alumnos, tienen una característica esencial que no poseen los grupos de alumnos adultos. No podemos olvidar que los adolescentes están en la escuela por obligación" de acuerdo con Martínez (2016, p. 58).

La propuesta que aquí presentamos está destinada a estudiantes brasileños entre 12 y 15 años que ya cursaron un nivel inicial de lengua española y poseen un nivel 
cercano a A2, de acuerdo con la clasificación de competencias descriptas en el Marco Común Europeo de Referencia para las lenguas (CONSEJO DE EUROPA, 2002).

El tema escogido se planificó y desarrolló a partir del interés de los alumnos en los intercambios estudiantiles. Esto también nos ayudó a perfilar el léxico ocasional que sustenta el objetivo principal del material, la enseñanza de las colocaciones de verbo+ sustantivo. Entendemos que las vivencias dentro y fuera del ambiente escolar abren un abanico de interacciones significativas que permiten aplicar, eficazmente, el enfoque léxico dentro de nuestra práctica pedagógica. Por ese motivo se elaboró esta actividad que trata de un intercambio estudiantil de una semana entre una escuela de Porto Alegre (Brasil) y una de Montevideo (Uruguay) en forma de novela, que también incluye la búsqueda de un tesoro de monedas de oro. Es un tema que conlleva información cultural para inducir al alumno a desarrollar, asimismo, su competencia sociocultural y, así, ayudarlo a conocer de forma constructiva otras culturas evitando estereotipos y prejuicios. Escribe Giovanni et al. (2000, p. 34) que si se desea que el alumno sea competente comunicativamente, se "deberá conceder un papel esencial al componente cultural, como algo indispensable e indisociable de la competencia comunicativa".

Elaboramos esta propuesta por medio de una pbwork ${ }^{1}$, aplicación en línea para la construcción de páginas web, gratuita para uso educativo, que permite el libre acceso a través de una dirección web. Este material presenta la ventaja de ser un espacio común, en línea, que puede compartirse digitalmente con todos los alumnos propiciando una labor colaborativa que, al mismo tiempo, estimula la autonomía. Pensando en que otros docentes podrían hacer uso de este material, como apoyo, también se elaboró una pbwork exclusiva para el profesor con orientaciones didácticas.

A seguir, presentamos la portada del material:

\section{LECTURA EN ESPAÑOL PARA EL APRENDIZAJE DE COLOCACIONES DIVERGENTES DE SUSTANTIVO Y VERBO ¡VIVAN LOS BIZCOCHOS Y LOS ALFAJORES!}

Flor se va de intercambio a Montevideo, capital de Uruguay y encuentra un tesoro. Con Flor descubrirás nuevas costumbres y practicarás las COLOCACIONES de verbo y sustantivo diferentes entre español y portugués.

Las COLOCACIONES son combinaciones de palabras que se usan juntas, algunas son IGUALES en español y en portugués como tener una entrevista $($ español $)=$ ter uma entrevista (portugués) pero otras son DIFERENTES como tener éxito (español) $\neq$ ter sucesso (portugués) tener clase (español) $\neq$ ter aula (portugués) 
Encontrarás las colocaciones diferentes del portugués en letra negrita y al pulsar este enlace se abrirá una ventana. De esta forma conocerás cómo se dicen en portugués y un ejemplo de cómo se usan.

Forma tu pandilla y descubre la historia de Flor.

Presta atención: cuando encuentres la sección PALABRAS ASOCIADAS, completa las tareas y gana puntos. El premio final es... Ya verás. Cada pandilla abrirá su propia pbwork para hacer las tareas.

Para entrar en la historia pulsa aquí ¡BUENA SUERTE!

La novela consta de nueve secciones que transcurren entre la selección de los alumnos para participar del intercambio y la preparación del viaje (primera sección) y cada uno de los ocho días (las ocho secciones restantes) que dura el intercambio. Cada sección presenta un conjunto entre diez y doce colocaciones de verbo+sustantivo, pues concordamos con Higueras García (2006, p. 110) cuando observa "que la mayoría de expertos en la enseñanza de léxico desaconsejan presentar y trabajar más de doce unidades léxicas al día”. El material corresponde a ocho clases de 45 minutos (estimado), sin embargo, esto depende del tiempo que establezca cada profesor.

Todas las colocaciones divergentes que se trabajan en el texto aparecen en negrita, como llamado de atención para el alumno, con la finalidad de que los alumnos las perciban, puedan procurar el hyperlink correspondiente y reparen en su equivalente en portugués. También se incluye una frase con la colocación en contexto para inferir su uso en la lengua española. El link permite volver al día o a los días en que aparece la colocación en el texto. La narrativa incluye otras colocaciones, además de aquellas que están en negrita; sin embargo, supeditado a una organización didáctica, se decidió focalizar el interés de los alumnos en las colocaciones divergentes destacadas en el material. Las notas, al pie de la página, aclaran el significado de algunas palabras o connotaciones culturales.

A continuación, una muestra de una sección:

Susy espera su turno y contesta: -Miraban las estrellas.

La profesora: - Muy bien, Susy. Magallanes y Colón navegaron guiándose por la Estrella del Norte y por ese motivo el norte pasó a ser referencia, pero no hay una explicación científica sobre qué parte se debe poner arriba en el mapa y qué parte se pone abajo. Es simplemente una convención. Y Torres García, por medio de este mapa, nos mostró que se puede ver el mundo desde varios ángulos, dándole otra forma.

Ese día me quedé pensando en las palabras de la profesora. Todo depende de la forma en que se miren las cosas. 
El profesor Gonzalo, de Idioma Español, nos dio una clase sobre el gaucho, símbolo importantísimo de la cultura rioplatense y sobre aspectos del folklore uruguayo como el pericón [2], la milonga [3] y la payada [4]. El tema me interesó y tomé apuntes de todo lo que el profe dijo. Descubrí que los gaúchos y los uruguayos tenemos mucho en común como tomar mate, algunas palabras de nuestro vocabulario, comer churrasco, el clima, ...

Al final de algunas de las secciones se presentan ejercicios variados contextualizados en los que se busca promover la práctica efectiva de las colocaciones destacadas en el texto. Por ejemplo, en una sección se presenta un juego con colocaciones ya trabajadas utilizando la herramienta Kahoot ${ }^{2}$ que permite gamificar, por medio de un cuestionario, un material determinado. Los alumnos realizan la tarea de forma digital en su pbwork.

A seguir, una de las tareas del material:

\section{PALABRAS ASOCIADAS}

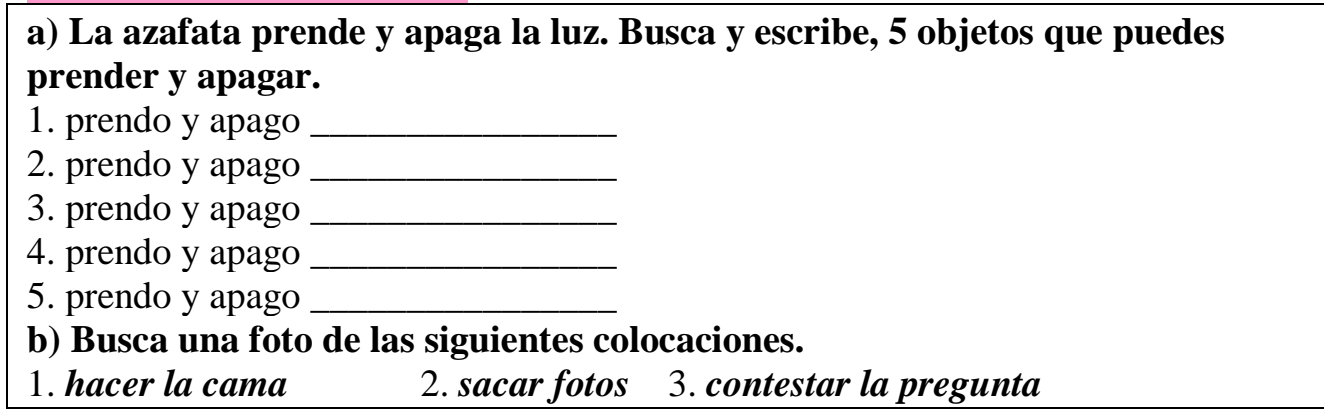

Para que los estudiantes realicen, de forma efectiva, las actividades solicitadas, adoptamos la estrategia de la gamificación que significa aplicar dinámicas que incluyen recompensa de puntos, tarjetas motivadoras, metas, etc. y que motivan al alumno por medio de una competición válida que incentiva el progreso de su aprendizaje. No se trata simplemente de quien concluyó más rápido, sino si lo hizo de forma completa, original o más elaborada. Los ejercicios se trabajan de forma cooperativa, en parejas. Las producciones escritas son en lengua española y el profesor sólo coordina las actividades y puede ir corrigiendo y evaluando los ejercicios de los alumnos, pues tiene acceso a todas las pbworks.

Como tarea final, los alumnos tienen que elaborar una historia multimodal en línea utilizando veinte de las colocaciones trabajadas a lo largo de la novela. Concordamos con Martínez (2016) que sugiere trabajar con los adolescentes por medio de tareas comunicativas entendidas como "tareas para aprender lengua haciendo cosas 
con palabras" y aclara que "en las tareas para aprender un idioma, la lengua se usa para describir, contar, redactar, discutir, imaginar y crear" (ídem, p. 60). De este modo, las tareas son un medio de alcanzar un objetivo final y para que la metodología con tareas funcione se debe "proponer un producto que sea tangible, visible, evaluable y compartible" (MARTÍNEZ, 2016, p. 61) como es en este caso. Consideramos que esta tarea representa una práctica comunicativa creativa, al mismo tiempo que se practican las colocaciones divergentes.

\section{PALABRAS ASOCIADAS: TAREA FINAL}

En esta historia ocurre un hecho insólito, Flor y sus amigos encuentran monedas de oro de un galeón del SXVII. Piensa con tu pandilla en un hecho insólito (poco frecuente o inverosímil) y escribe una historia corta incluyendo veinte de las colocaciones que aprendiste en esta novela. Tu profesor te ayudará con recursos y herramientas de internet. El formato es libre, puedes crear un comic en viñetas, un fanzine, un álbum, etc. Todos ganarán puntos y al final habrá un vencedor.

\section{CONCLUSIONES}

En el contexto de enseñanza de ELE en Brasil, es de suma importancia el desarrollo de la competencia léxica colocacional con el fin de que los estudiantes brasileños, por medio de una concientización sobre este fenómeno, un aprendizaje explícito de las colocaciones más relevantes y una práctica variada, puedan superar esta dificultad mejorando así su competencia comunicativa.

Este material didáctico en línea está dirigido a las necesidades e intereses de un público meta de aprendices brasileños pertenecientes a la Enseñanza Básica (12-16 años), de nivel cercano a A2. Esta propuesta producida por medio de una pbwork acompaña la relevancia de las competencias digitales en la realidad de los estudiantes.

La enseñanza de ELE no es estática, los elementos que la constituyen: los estudiantes, la lengua, el aprendizaje, la enseñanza son dinámicos, y esto representa, en definitiva, nuevas posibilidades didácticas. La evolución digital aporta herramientas que deben utilizarse en el aprendizaje de una lengua extranjera como forma de motivar a los alumnos a aprender con estímulos interesantes y que llamen su atención. La escuela debe ser receptiva a estos cambios y modificar sus técnicas de enseñanza-aprendizaje. Por otra parte, es imprescindible tomar en cuenta los intereses de los alumnos, frecuentemente conectados a la red, para elaborar las actividades didácticas. 
Estamos continuamente buscando la mejor forma de enseñar y de que los alumnos, en definitiva, aprendan y vislumbramos que la senda para mejorar nuestras prácticas educativas incluye las TIC. Pujolà (2016) entiende que la motivación de los jóvenes al usar las TIC en el aula ya no se debe a la novedad, sino al tipo de propuesta de tarea que debe incluir "un carácter lúdico o novedoso para que motive a los adolescentes en el aprendizaje de ELE" (ídem, p. 125). Las nuevas formas de interlocución entre los estudiantes nos llevan a reflexionar e innovar adoptando las TIC en la metodología de nuestras clases de ELE.

\section{Referências}

BOSQUE, Ignacio. REDES Diccionario combinatorio del español contemporáneo: las palabras en su contexto. Madrid: Ediciones SM., 2010.

CASSANY, Daniel. En_línea Leer y escribir en la red. Barcelona: Editorial Anagrama, 2012.

CONSEJO DE EUROPA. Marco común europeo de referencia para las lenguas: aprendizaje, enseñanza y evaluación. Traducción: Instituto Cervantes. Madrid: Ministerio de Educación, Cultura y Deporte, 2002. Disponible en: http://cvc.cervantes.es/ensenanza/biblioteca_ele/marco/indice.htm. Consulta: 20 jul. 2020.

CORPAS PASTOR, Gloria. Manual de fraseología española. Madrid: Gredos, 1996.

COSERIU, Eugenio. Lições de Linguística Geral. Traducão de BECHARA, Evanildo. Rio de Janeiro: Ao Livro Técnico, 1980.

DUDENEY, Gavin; HOCKLY, Nicky; PEGRUM, Mark. Letramentos digitais. Traducção de MARCIONILO, Marcos. São Paulo: Parábola, 2016.

FERNÁNDEZ LÓPEZ, Sonsoles. Enfoque de acción: Aprender lenguas con "Tareas". In: LLUCH ANDRES, Antoni; FERNÁNDEZ LÓPEZ, Sonsoles; NAVARRO BLANCO, Antonia. (eds.). Enfoque por tareas: Propuestas didácticas. Colección Complementos. Serie Didáctica, Embajada de España en Brasil, Conserjería de Educación, 2010, p. 5-19.

GARCÍA SALIDO, Marcos. La frecuencia de corpus como criterio para nivelar colocaciones léxicas. Dossier thématique: Corpus y diccionarios para la investigación en lengua española. Études romanes de Brno, Brno, v. 38, n. 2, 2017.

GIOVANNI, Arno et al. Profesor en acción 2 Áreas de trabajo. Madrid: Edelsa, 2000. 
HIGUERAS GARCÍA, Marta. Estudio de las colocaciones léxicas y su enseñanza en español como lengua extranjera. Colección Monografías, Málaga: ASELE, Secretaría General Técnica, n. 9, 2006.

ÍRSULA, Jesús. Entre el verbo y el sustantivo, ¿quién rige a quién? El verbo en las colocaciones sustantivo-verbales. In: ENDRUSCHAT, Annette; VILELA, Mario; WOTJAK, Gerd (eds.). Verbo e estruturas frásicas. Colóquio Internacional de Linguística Hispânica de Leipzig, IV, 1993. Actas [...]. Porto: Revista da Faculdade de Letras da Universidade de Porto, v. 6. 1994, p. 277-286.

KOIKE, Kazumi. Colocaciones léxicas en el español actual: estudio formal y léxicosemántico. Madrid: Universidad de Alcalá. 2000.

MARTÍNEZ, Matilde. Tareas con adolescentes, sí o sí. In: HERRERA, Francisco; SANS, Neus. Enseñar español a niños y adolescentes. Enfoques y tendencias. Barcelona: Difusión, 2016, p. 55-65.

MOREIRA KENSKI, Vani. Tecnologias e ensino presencial e a distância. Campinas: Papirus, 2008.

PUJOLÀ, Joan-Tomàs. Niños y adolescentes movilizados y enredados en el aprendizaje de español. In: HERRERA, Francisco; SANS, Neus. Eds. Enseñar español a niños y adolescentes. Enfoques y tendencias. Barcelona: Difusión, 2016, p.186-195.

REAL ACADEMIA ESPAÑOLA. Corpus del Español del siglo XXI (CORPES). Disponible en:

http://web.frl.es/CORPES/org/publico/pages/colocacion/coaparicion.view. Consulta: 20 jul. 2020.

RICHARDS, Jack; RODGERS, Theodore. Enfoques y métodos en la enseñanza de idiomas. Traducción de CASTRILLO, José Manuel. Madrid: Cambridge University Press, 1998.

SÁNCHEZ RUFAT, Anna. Apuntes sobre las combinaciones léxicas y el concepto de colocación. Anuario de Estudios Filológicos, v. XXXIII, 2010, p. 291-306.

\footnotetext{
${ }^{1}$ http://www.pbworks.com/

2 https://kahoot.com/
} 